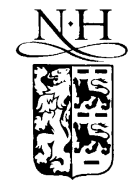

ELSEVIER

\title{
Surface induced nucleation of the helifan phase
}

\author{
V.D. Mello ${ }^{\text {a }}$, A.S. Carriço ${ }^{\text {b,* }}$ \\ a Departamento de Física, Universidade do Estado do Rio Grande do Norte, Mossoró-RN 59610-210, Brazil \\ b Departamento de Física, Universidade Federal do Rio Grande do Norte, Natal-RN 59072-970, Brazil
}

\begin{abstract}
We calculate the magnetic phases of thin films of helimagnetic materials. The surface of the film is perpendicular to the $c$-axis so that, for $H=0$, the moments are in the basal plane. We study the effect of external fields, applied in the basal plane, and find that the field effects are controlled to a large extent by the $c$-axis anisotropy. If the $c$-axis anisotropy is small, the effect of the external field is to produce small modifications in the basal plane projections, which remain nearly in a helical pattern, while the $c$-axis components oscillate throughout the film, with the surface regions displaying stronger oscillations. By increasing the external field strength, this phase evolves to a first order transition to a non-uniform fan phase, with major modifications near the surfaces. If the $c$-axis anisotropy is strong the out of plane oscillations are inhibited, and depending on the film thickness, a surface helifan phase is nucleated, starting from the gradual alignment of the magnetic moments near the surface region with the external field. The phase has twice the period of the helix and nucleates only in films where the thickness fits the double-period structure. () 2001 Elsevier Science B.V. All rights reserved.
\end{abstract}

Keywords: Computer simulations; Low index single crystal surfaces; Lanthanides

\section{Introduction}

The development of techniques to grow metallic structures has renewed the interest in the study of the magnetic structure of rare earth (RE) materials. A great deal of research effort has recently been dedicated to the study of magnetic multilayers made of RE films interleaved with films of RE or non-RE materials [1-3].

The magnetic properties of thin helimagnetic films, under applied field, have been explored theoretically [4] and it was shown that new phases

\footnotetext{
${ }^{*}$ Corresponding author. Fax: +55-84-2153-791.

E-mail address: acarrico@dfte.ufrn.br (A.S. Carriço).
}

are induced by the reduced coordination of the spins near the surfaces of the films. In this study thin films of Ho and Dy were considered.

Early in the last decade new magnetic phases of helical materials have been discovered [5]. These phases, called helifans, consist of the combination of known phases, the helix and the fan phase. In the helical phase the moments of successive planes are turned by a constant angle, while in the fan phase the moments oscillate around the field direction. In the helifan phases there a number of planes which follow the helical arrangements and interact with groups of planes where the moments oscillate around the applied field with small amplitudes, like in the fan phase.

This is a case of the coexistence of different magnetic phases. There are theoretical reports of 
mixed phases near the surfaces of antiferromagnetic (AF) films [6] and also in AF films and multilayers made of transition metal films [7,8]. These magnetic phases consist of the coexistence of the AF phase and the spin flop (SF) phase. The mixed phase, called surface spin flop (SSF), is nucleated at the surfaces by an applied field parallel to the surface and opposite to surface spins.

The SSF phase results from the existence of softer spins near the surfaces. In the SSF phase the near surface region is in the SF phase while most of the AF film (or AF multilayer) is in the AF phase. The instability of the AF phase occurs at a value of the external field strength which is smaller than that which would produce the instability of the AF phase in the bulk. For this reason the spins far away from the surface of the AF system tend to stay in the AF state. The SSF phase has been observed in $\mathrm{Fe} / \mathrm{Cr}$ multilayers with an even number of magnetic layers [9].

In AF films and multilayers the transition to the SSF phase is accompanied by a jump of magnetization, characteristic of a first order phase transition [8]. Therefore no modification in the AF order is possible if the external field strength is smaller than the threshold value of AF instability. The effect of a weak external field applied in the basal plane of helimagnetic materials is to produce small modifications in the helix [10], inducing a small magnetization in the field direction. The distortions in the helical structure increase with the strength of the external field and only after a large value of $H$ a first order transition to the fan phase occurs.

The near surface region of a RE thin film has a reduction of the exchange coupling. Thus one might expect field-induced modifications in the helical order to start in the near surface region. It thus seems plausible to expect the helifan phase to be favored by surface effects. The argument is similar to that which applies to AF systems, since the spins of the surface region of thin films are more easily turned towards the field direction.

In this paper we report a study of the influence of surfaces in the nucleation of the helifan phase in thin helimagnetic films. In Section 2 we describe the theoretical model, in Section 3 we discuss the magnetic patterns induced by external field and in the final section we present our conclusions.

\section{Local effective field model}

We consider exchange energy, Zeeman energy and an anisotropy energy which holds the magnetic moments in the basal plane. No in-plane anisotropy energy is included, as appropriate to moderately high temperatures. The magnetic energy is given by:

$$
\begin{aligned}
E= & J_{1} \sum_{n=1}^{N-1} \vec{S}(n) \cdot \vec{S}(n+1)+J_{2} \sum_{n=1}^{N-2} \vec{S}(n) \cdot \vec{S}(n+2) \\
& +\sum_{n=1}^{N}\left\{D S_{z}^{2}(n)-\gamma \vec{S}(n) \cdot \vec{H}_{0}\right\}
\end{aligned}
$$

$J_{1}$ and $J_{2}$ are the parameters that describe the exchange interaction between the nearest and next nearest spins respectively, $\vec{S}(n)$ denotes spins in the $n$th monolayer, $D$ is the parameter that specify the anisotropy, holding the moments in the basal plane, $\gamma=g \mu_{\mathrm{B}}$, and $\vec{H}_{0}$ is the applied dc field in the basal plane. Throughout the paper $D$ is written in units of $J_{2}$.

Surface effects are incorporated in Eq. (1) since the spins near the surfaces have the exchange energy reduced by the absence of nearest and second nearest neighbors. Therefore only the spins of the first two planes near the surfaces $(n=1,2, N-1$ and $N$ ) are directly affected by surface effects. However the lack of coordination near the surfaces may be felt by spins deep inside the film. The helix as a whole accommodates to the applied field and surface effects.

We consider that $|\vec{S}(n)|=S$ is a constant, so that we can write the components of $\vec{S}(n)$ as $S_{x}(n)=S \sin \left(\theta_{n}\right) \cos \left(\varphi_{n}\right), S_{y}(n)=S \sin \left(\theta_{n}\right) \sin \left(\varphi_{n}\right)$ and $S_{z}(n)=S \cos \left(\theta_{n}\right)$, where the angles $\theta_{n}$ and $\varphi_{n}$ determine the direction of $\vec{S}(n)$ with respect to the $z$-axis, the direction of the normal to the surface, and the angle of the projection of $S(n)$ in the basal plane with the $x$-axis (in-plane) respectively. The external field is along the $x$-axis.

The equilibrium configuration is obtained from the angles $\left[\left(\theta_{n}, \varphi_{n}\right), n=1, \ldots, N\right]$ that minimize the magnetic energy by Eq. (1). The numeric algorithm is equivalent to looking for values of $\theta_{n}$ and $\varphi_{n}$ that make the torque on every $\operatorname{spin} \vec{S}(n)$ equal to zero. Therefore, to find out the equilibrium 
configuration we obtain the profile $\left\{\theta_{n}, \varphi_{n} ; n=1\right.$, $\ldots, N\}$, that makes $\vec{S}(n) \times \partial E / \partial \vec{S}(n)=0$ for all spins. The numerical method is described in more detail in Refs. $[7,8]$.

We focus on the differences of the field effects according to the strength of the anisotropy and exchange energies. For this purpose we study a helimagnetic model system, with a helix period of twelve planes, corresponding to a turn angle of $30^{\circ}$. We have examined the magnetic phases for values of $D$ ranging from very small to large. This corresponding to the strong temperature dependence of $D$ in real systems ( $D_{y}$ and $H_{0}$, for instance) within the helimagnetic phase.

Our results indicate that the magnetic behavior of the films is governed by the value of $D$ relative to the values of the exchange energy. We show that for small $D$ values the field effects in the film are similar to those in the bulk. However, if $D$ is large then the surface and field effects combined lead to the nucleation for the helifan phase.

\section{Field effects}

In thin films the magnetic energy density is not uniform. Near the surfaces there are considerable reduction of exchange energy. This might lead to modifications both in the helical order of the projection of the moments in the basal plane and also in the polar angle profile. Since the first neighbor exchange is ferromagnetic one may expect to find smaller angles between the moments in successive planes in the surface region. This lack of exchange near the surfaces may well be accommodated by allowing out of plane components. Thus if the $c$-axis anisotropy is small one might find large oscillations in the polar angle near the surfaces.

For small values of $D$ the out of plane components of the spins involve small values of the anisotropy energy. In this case for a small value of $H$ the fan phase is formed. However, if the $c$-axis anisotropy energy is increased the fan phase does not form for any value of the applied field. Instead, in the near surface region, where the coordination is reduced, the moments align with the applied field more strongly.

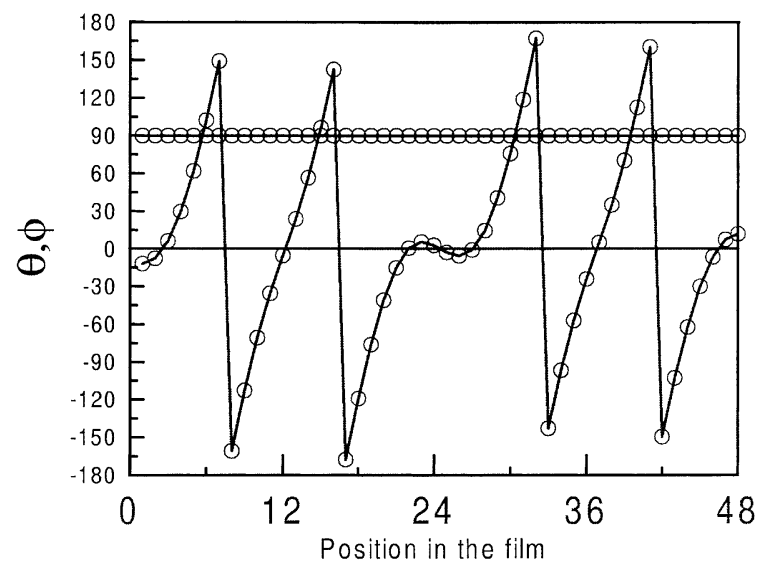

Fig. 1. The helifan phase induced by applied field in the basal plane. We show the magnetic pattern of a 48 atomic planes film, showing the angle of the projection in the basal plane and the polar angle.

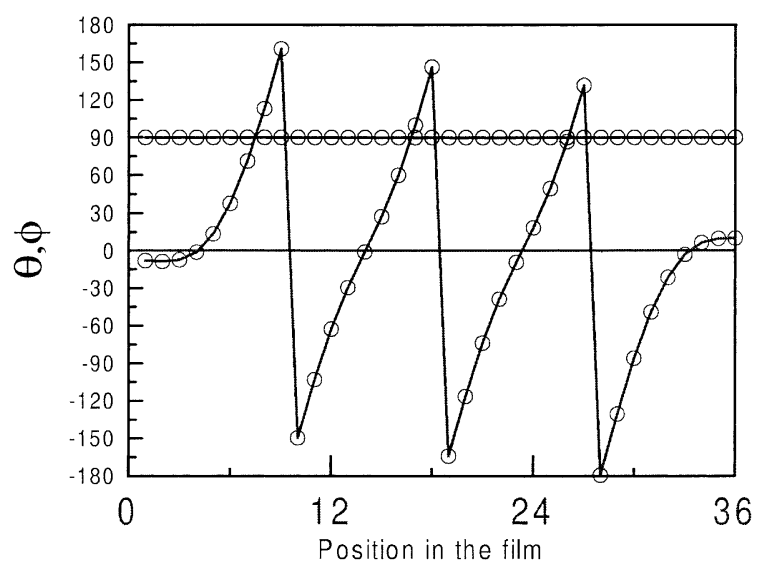

Fig. 2. Magnetic pattern of a 36 atomic planes film, showing the angle of the projection in the basal plane and the polar angle. The moments in the near surface region are aligned with the applied field.

Thickness effects may inhibit the formation of helifans. We have found that the helifan structure of thin films has twice the helix period. For thin films, near the surfaces the arrangement of spins look like the fan phase and the middle is the helical (deformed) state. Only beyond a certain thickness we have found the formation of helifans.

In Figs. 1 and 2 we show the angular profile for films with 36 and 48 atomic planes and $D=0.1$. These profiles have been calculated with the same 
value of the applied field strength. The 48 atomic planes film display a helifan with a period of 24 atomic planes while in the 36 atomic planes film the field effects are restricted to the surface regions. In both cases the field induced modifications of the helical structure does not produce any variation in the polar angle, fixed at the value of 90 , as imposed by the $c$-axis anisotropy.

The evolution of the magnetization of these two films follows different processes. The field effects in the 36 atomic planes film is to produce surface regions which gradually align with the applied field. The surface regions also become thicker as the applied field strength is increased. At the saturation field the whole film becomes aligned with the field direction.

In the 48 atomic planes film the field induced modifications of the helical structure occurs both near the surfaces and in the middle of the film. The deformation of the helix eventually leads to the formation of a helifan, with a period twice as large as that of the helix. As the applied field strength is increased the helifan locks in, keeping fan-like regions which gradually align with the field, and helical-like regions which are slightly modified by field effects.

When the anisotropy is small the magnetization process is different. In this case the moments os-

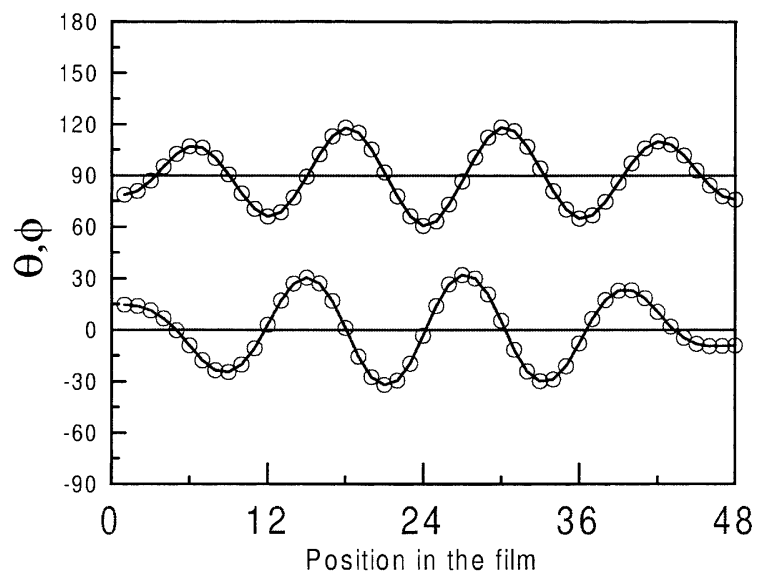

Fig. 3. Magnetic pattern of a 48 atomic planes film, for $D=0.001$, showing the angle of the projection in the basal plane and the polar angle. This pattern is the fan phase with large oscillations of the polar angle.

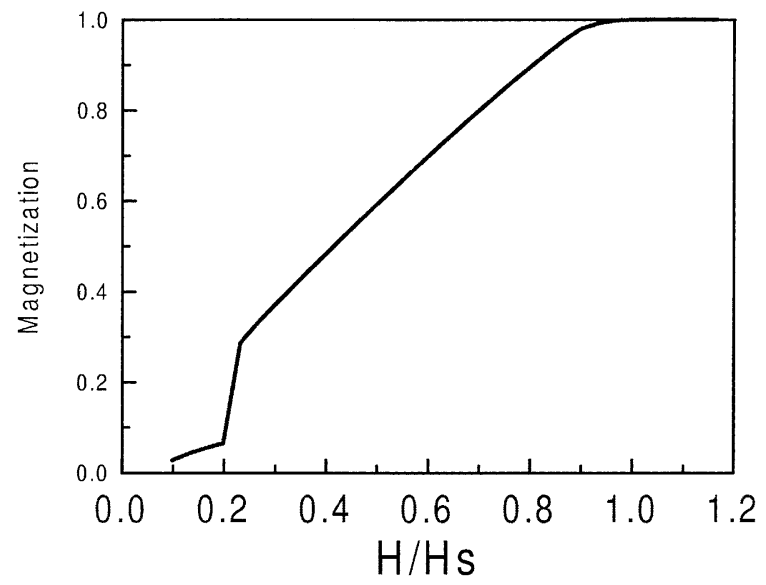

Fig. 4. Magnetization 48 atomic planes film for $D=0.001$. The discontinuity in the magnetization indicates the formation of the fan phase, modified by surface effects. The magnetization and applied field are shown in units of the saturation values.

cillate out of the basal plane and a bulk-like fan phase is nucleated at a small field value. In Fig. 3 we show the profile for the 48 atomic planes films with $D=0.001$. In this case it is seen that the fan phase covers the whole film. It is also seen that there are relevant oscillations of the value of the polar angle around the mean value of 90 .

In all the cases described above (Figs. 1-3) the profiles shown correspond to the applied field when the modified helix becomes unstable and a new magnetic phase sets in.

In Fig. 4 the magnetization jump occurs at a field value $H=0.2 H_{\mathrm{s}}$ and marks the onset of the fan phase in the whole film. $H_{\mathrm{s}}$ is the strength of the external field which saturates the magnetization. This phase is characterized by large oscillations in the polar angle, taking the moments out of the basal plane, while the projection of the moments in the basal plane oscillates around the applied field direction. A typical pattern is shown in Fig. 3.

In Fig. 5 the magnetization increases by field induced deformation of the helix. The moments keep in-plane $(\theta=\pi / 2)$ and the gradual deformation in the helix leads to the formation of the helifan phase.

The nucleation of the helifan does not represent a large change in the magnetization because in the 


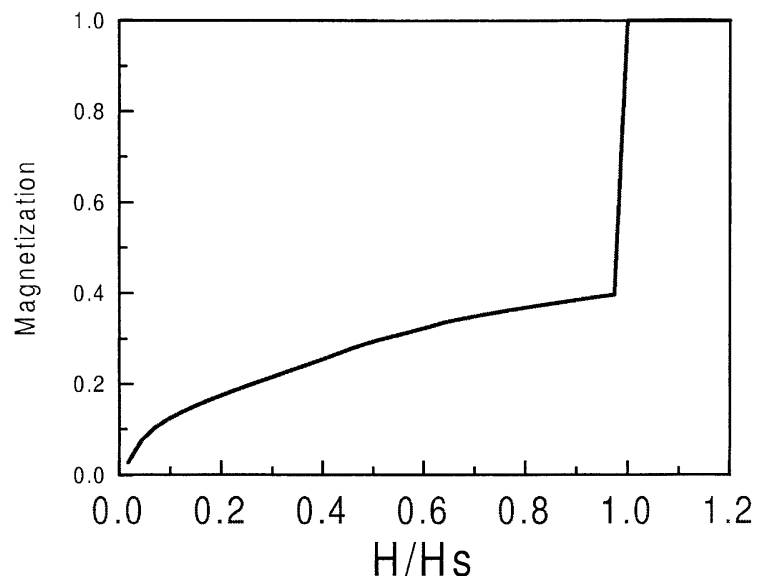

Fig. 5. Magnetization of a 48 atomic planes film for $D=0.1$. The discontinuity in the magnetization occurs for the value of field which produces saturation of the magnetization. The magnetization and applied field are shown in units of the saturation values.

helifan phase most of the moments follow a "deformed helix" pattern. The helifan locks in and only for large field values there is a discontinuous change of magnetization to the saturation value.

\section{Final remarks}

We have shown that surface effects favor the formation of helifans in helimagnetic films. As a result of the reduced coordination, the field can more easily turn the spins in the surface regions. This is the seed for the formation of the helifan structure if the helix is stabilized by the $c$-axis anisotropy.

One might expect no effect of $D$ while discussing field effects if the field is applied in the basal plane. This, however, is not the case. We have found that the saturation field increases if the value of $D$ is large. The modifications in helical pattern occurs via the accommodation of the structure to field and surface effects. For large values of $D$ there are no oscillations in the polar angle and the stability of the helix is significantly increased.

The existence of helifan structure is not easily detectable from the magnetization curve. In Fig. 5 we display the magnetization curve for a 48 atomic planes film where the helifan is formed for large fields near the saturation field. The magnetization curve of thinner films have also been calculated. The curves for films with 12, 24 and 36 atomic planes are very nearly the same as that shown in Fig. 5. Thus the detection of the helifan requires methods, such as neutron scattering, which can see details of the magnetic pattern. One key feature in this respect is that the helifans have a period different from that of the helix. In our present case the helifan has a period equal to twice the helix period.

\section{Acknowledgements}

We thank the Brazilian Research Council CNPq for partial financial support.

\section{References}

[1] J.P. Goff, R.S. Saethour, D.F. McMorrow, F. Yakhou, A. Stunault, R.C.C. Ward, M.R. Wells, Physica B 283 (2000) 180.

[2] J.P. Goff, R.S. Saethour, C. Micheletti, S. Langridge, C.J.T. Wilkins, R.C.C. Ward, M.R. Wells, J. Magn. Magn. Mat. 198 (1999) 309.

[3] D.A. Jehan, D.F. McMorrow, R.A. Cowley, C.C. Ward, M.R. Wells, N. Hagman, Phys. Rev. B 48 (1993) 5594.

[4] V.D. Mello, A.S. Carriço, N.S. Almeida, Phys. Rev. B 59 (1999) 6979.

[5] J. Jensen, A.R. Mackintosh, Phys. Rev. Lett. 22 (1990) 2699.

[6] D.L. Mills, Phys. Rev. Lett. 20 (1968) 18.

[7] A.S. Carriço, R.E. Camley, R.L. Stamps, Phys. Rev. B 50 (1994) 13453.

[8] A.L. Dantas, A.S. Carriço, Phys. Rev. B 59 (1999) 1223.

[9] R.W. Wang, D.L. Mills, E.E. Fullerton, J.E. Mattson, S.D. Bader, Phys. Rev. Lett. 72 (1994) 920.

[10] T. Nagamiya, J. Appl. Phys. 33 (1962) 1029. 